\title{
DUS Testing of Sesame (Sesamum indicum L.) Accessions Using Morphological Descriptors and Evaluation for Foliar Diseases of Sesame
}

\author{
M. G. Palakshappa ${ }^{1}$, Harshiya Banu' ${ }^{2}$, S. G. Parmeshwarappa ${ }^{3}$, \\ Rajani Bisen ${ }^{* *}$, H. Nagappa ${ }^{5}$ and Pooja Holeyannavar ${ }^{6}$ \\ All India Co-ordinated Research Project on Sesame and Niger, MARS, UAS, Krishinagar, \\ Dharwad, India \\ *Project Co-ordinating Unit, Sesame and Niger, JNKVV, Jabalpur, India \\ *Corresponding author
}

\section{Keywords}

DUS testing, Characterization, Accessions, Sesame, Cercospora, Bacterial leaf blight

Article Info

Accepted: 15 December 2019 Available Online: 20 January 2020

\section{A B S T R A C T}

The aim of the present study is to characterize Seven hundred and twenty accessions of Sesame (Sesamum indicum L.) along with two National check varieties viz., GT-10, TKG22 and local check variety DS-5 were characterized based on the DUS descriptors. The experiment was executed in Augmented Block Design without replication at All India Coordinated Research Project on Sesame and Network on Niger experimental plot, Main Agricultural Research Station, University of Agricultural Sciences, Dharwad, Karnataka during kharif 2016. On the basis of DUS descriptors, Sesame accessions were characterized for twenty morphological descriptors and grouped into five DUS traits, viz. Growth, Stem, Leaf, Inflorescence and Capsule characters and evaluated for foliar diseases. A significant amount of variation was observed for most of the traits studied. Study of growth traits clearly revealed that majority of the genotypes showed variability for growth habit towards semi-erect types with determinate growth types and sparse hairiness on stem with majority of basal branching pattern. Towards stem characters, higher frequency of sparse/weak hairiness, lanceolate leaf shape for middle leaves, linear leaf shape for top leaves, entire basal leaf margin, absence of lobe incision of basal leaf and green petiole colour for leaf characters was observed in the studied set, respectively. Regarding inflorescence descriptors, $86.7 \%$ of accessions exhibited one flower/leaf axil. Flower interior and exterior colour 38-39\% respectively. Sparse type corolla hairiness was in majority. Similarly, four number of locules/capsule, narrow oblong bicarpellate capsule shape, monocapsular arrangement, glaborous type of capsule hairiness for capsule characters also in higher frequency. 26.3 per cent of tested accessions have recorded beige colour at post harvest stage. Among 720 accessions studied only $1.3 \%$ of accessions rated grade 2 for bacterial leaf blight and $9.3 \%$ of accessions scored grade 3 for Cercospora leaf spot indicating moderately resistant genotypes. The identified DUS traits will serve as a marker in selection process which are less influenced by environmental fluctuations and are easily scorable and stable expression. They serve as diagnostic descriptors of germplasm accessions and hence useful to avoid mistakes in labelling, aid identification and minimize duplication in the germplasm and helps in the development of guidelines for varietal release and registration of new varieties of sesame. The study revealed the distinct characteristics of sesame accessions for morphological variations that exist in these lines due to variation in genetic makeup and could be better utilized by breeders in the selection based on their specific requirement for breeding programme. This study is highly useful for varietal identification and conservation. 


\section{Introduction}

Sesame (Sesamum indicum L.), a member of the order Tubiflorae belongs to family Pedaliaceae is an ancient oil seed known to humankind, sesame seeds have been widely employed in culinary as well as in traditional medicines for their nutritive, preventive, and curative properties, (Weiss, 1971). It is an important annual oilseed crop in the tropics and warm subtropics, where it is usually grown in small plots (Bedigian and Harlan, 1986). Owing to its high quality, Sesame is also referred to as the "Queen of oil seed crops" (Deepthi et al., 2014) because it contains high oil (38- 54\%), protein (18$25 \%$ ), calcium, phosphorous, oxalic acid and excellent qualities of seed oil and meal (Prasad, 2002). Sesamum seed oil has long shelf life due to the presence of lignans viz., Sesamin, Sesaminol and Sesamolinol which have remarkable antioxidant function, resisting the oxidation. They are highly valued for their oil which is exceptionally resistant to rancidity. "Open sesame" the famous phrase from the Arabian Nights reflects the distinguishing feature of the sesame seed pod, which bursts open when it reaches maturity. Characterization should eventually lead to a system of recording and storing useful data that can be readily retrieved and made available to others and help in planning breeding programmes (Debas et al., 1994). Among the several limiting factors for successful sesame production, yield loss due to diseases is one of the major constraints. Sesame suffers from many foliar diseases viz. Cercospora leaf spot, Powdery mildew, Alternaria and bacterial leaf blight. Germplasm forms the raw material for any crop improvement programme. There is wide genetic diversity available in sesame and characterizing these resources is a prerequisite for the genetic improvement of its cultivars. The characterization and evaluation are the important pre requisites for effective utilization of germplasm and also to identify sources of useful genes (Upadhayay et al., 2010). In order to introduce a new plant variety to the markets commercially, it is necessary to register newly bred variety, which relies upon the results of DUS (distinctness, uniformity and stability) tests for a new genotype to be registered as a commercial variety, it needs to be distinct from all other released varieties, uniform and stable for morphological and other evaluated traits (Lombard et al., 2000 and Tommasini et al., 2003). Therefore, DUS test has been established to be the foundation of plant variety protection and also to identify a new variety from reference collection (Kwon et al., 2005). There are several issues to be resolved with yield and yield attributing traits like seed yield, frequency of shattering and oil content. Ideotype breeding aimed at modifying the plant architecture is also timetested strategy to increase the yield potential. Therefore, the present study was undertaken to characterize the accessions using DUS descriptors in sesame.

\section{Materials and Methods}

Seven hundred and twenty accessions of Sesame along with two National check varieties viz., GT-10, TKG-22 and DS-5 as Local check were grown using unreplicated augmented block design (Federer, 1956) at All India Co-ordinated Research Project on Sesame and network on Niger experimental plot, Main Agricultural Research Station, University of Agricultural Sciences, Dharwad, Karnataka during Kharif 2016. The distance between rows was maintained at 30 $\mathrm{cm}$ and plant to plant $10 \mathrm{~cm}$. The crop was raised under recommended package of practices along with prophylactic protection measures. The recommended dose of fertilizer was 50:25:25 NPK Kg/ha. The basal dose of 25:25:25 NPK Kg/ha was applied at the time of sowing and remaining $25 \mathrm{~kg}$ of nitrogen 
was applied 30 days after sowing as top dressing. The plants were thinned out 20-25 days after sowing leaving a single healthy seedling at a distance of $10 \mathrm{~cm}$ per hill. The crop was kept weed free and three hand weedings were carried out during the crop growth period. Necessary plant protection measures were taken to control pest and disease. Due to severe Bacterial leaf blight, Powdery mildew and Cercospora leaf spot diseases during flowering to capsule formation stage, $\mathrm{COC}(0.2 \mathrm{~g})+$ Streptocyclin $(0.5 \mathrm{~g})$ per litre of water was sprayed to control Bacterial leaf blight and Carbendizim @ 0.1 per cent was sprayed for the effective control of Cercospora leaf spot (Palakshappa et al., 2009)

The observations were recorded on plant growth type, plant growth habit, Stem hairiness and branching pattern as plant descriptors. Leaf hairiness, leaf shape, basal leaf margin lobe incision of basal leaf and petiole colour as leaf descriptors.

Number of flowers per leaf axil, Corolla hairiness, Exterior corolla colour and Interior corolla colour as inflorescence descriptors. No. of locules per capsule, Bicarpellate capsule shape, Capsule arrangement, Capsule hairiness, Colour of dry Capsule (Sun dried) and Type of capsule break as Capsule descriptors with Seed coat colour. Also recorded plant height, number of branches, number of capsules, test weight along with screening for bacterial leaf blight and Cercospora leaf spot resistance in the present study according to 0-5 scale (Anonymous, 2015) for recording Cercospora and bacterial leaf blight disease score adapted by Project Co-ordinating Unit, All India Coordinated Research Project on Sesame and Niger (Indain Council of Agricultural Research) JNKVV Campus, Jabalpur, Madhya Pradesh, India.

\section{DUS Testing}

Twenty morphological descriptors have been considered essential for the description of Seven hundred and twenty accessions of sesame using guidelines for the conducting test for distinctiveness, uniformity and stability in sesame according to Descriptors for Sesame (Sesamum spp.) 2004 developed by IPGRI Rome, Italy and NBPGR New Delhi, India and data analysis for frequency distribution was analysed by Statistical Package for Social Science (SPSS).

\section{Results and Discussion}

The germplasm accessions under study showed wide range of variability for all the qualitative traits studied. Twenty qualitative traits were recorded for seven hundred and twenty accessions along with national checks GT-10 and TKG-22 and local check DS-5. The accessions were characterized based on the variability present in each one of the qualitative traits under different sub descriptors. The results for each trait are described briefly presented in Table 1 and its graphical representation is shown in Figure $1 \mathrm{a}, 1 \mathrm{~b}, 1 \mathrm{c}$ and quantitative characters in Table 2. Characterization at different growth stages of crop were discussed below.

\section{Plant descriptors}

Depending on the plant growth type, the accessions were classified into determinate and indeterminate. Majority of genotypes exhibited determinate $(63.1 \%)$ and $36.9 \%$ exhibited indeterminate growth type. In case of plant growth habit higher frequency of semi-erect $(42.7 \%)$ with sparse stem hairiness of $52.3 \%$ followed by dense hairs on stem with $20.7 \%$.

Similar results were reported by (Bhagwat Singh et al., 2017) in Sesame and (Harshiya 
Banu et al, 2018) in foxtail millet. Hairiness and pubescence is reported in effective for conferring resistance to insect pests in Dolichos bean (Jagadeesh Babu et al., 2008). Basal and top branching pattern exhibited to an extent of $52.6 \%$ and $38.8 \%$, respectively. Hairiness is the significant character for improving more seed yield and natural defense mechanism for biotic and abiotic factors. So this character may be recognized as ideal plant type. In the present study basal and top branching patterns were observed and it was indicated that the inheritance of branching habit was determined by one single dominant gene. However genetic basis of them has remained elusive. Similar findings have been reported in Sesame by Sarita et al. (2013).

\section{Leaf descriptors}

Only $6 \%$ of accessions exhibited profuse leaf hairiness followed by $22.4 \%$ of medium hairiness accessions indicating absence of bristles or pubescence containing silica can significantly enhance insect pest resistance in plants with consequent yield increases according to Laing et al. (2006) in poaceae family. Leaf shape revealed $32.4 \%$ and $64 \%$ of lanceolate and linear leaf shape respectively. Similar results were observed by Kashiram (1930) in Sesame. Majority of accessions are entire type basal leaf margin $(45.8 \%)$ whereas dentate and serrate revealed with a frequency of $34.2 \%$ and $20.0 \%$ respectively. Similar report was observed by Suhasini (2006) in Sesame. With respect to lobe incision of basal leaf most of genotypes do not have lobes on basal leaf and only 2.4 $\%$ accessions recorded strong lobe incision on basal leaf. About $99.2 \%$ of genotypes recorded green petiole colour at peak flowering stage did not show variability among accessions due to low intensity of anthocyanin pigmentation which is governed genetically and also influenced by the light intensity and nutritional status of the soil under which the crop is raised. Similar results were also reported by Ezilkumar (1999) and Jain et al. (2002). At maturity indicating that high yield was associated with purple pigmentation in plant parts was reported by Shigeta (1985) in finger millet. These findings are in consonance with those of Harshiya Banu et al., (2018)

\section{Inflorescence descriptors}

Only $13.3 \%$ of genotypes have more than one flower per leaf axil and sparse type corolla hairiness was observed up to an extent of 38.9 $\%$. Regarding exterior and interior corolla colour, both exhibited white colour (39\% and $38.8 \%)$ of total accessions respectively followed by white with pink shading $(27.2 \%$ and $26.0 \%$ ) and purple colour is just $0.1 \%$. This observation in Sesame is in accordance with Suhasini (2006) and Bhagwat Singh et al. (2017). Similar study reported in Foxtail millet by Harshiya Banu et al. (2018) indicating non-pigmented inflorescence was represented in higher frequency than those with pigmented inflorescence accessions.

\section{Capsule descriptors}

Majority of accessions observed to be four locules per capsule $(95.7 \%)$ and $1.4 \%$ of six and eight locules per capsule, but four locules are preferred by the breeders due to fertile seed whereas, six and eight locules per capsule are not selective due to chaffy seeds. Similar findings have been reported by Bhagwat Singh et al. (2017). Regarding shape of capsule $47.6 \%$ of accessions exhibited narrow oblong bicarpellate capsule shape and $94.4 \%$ are monocapsular arrangement of capsules. Capsule hairiness $(11.5 \%)$ are strong or profuse. Brown/tan colour of dry capsule $(49.3 \%)$ and $41.5 \%$ are yellow/straw colour. Majority of long type of capsule break was $29.0 \%$ and short type break was 
observed to be $26.5 \%$. The present results are in consonance with observations of Bhagwat Singh et al. (2017) and Suhasini (2006) suggesting short capsule break with less shattering reflects in yield increase and farmers preference.

Table.1 Morphological characterization of germplasm for descriptor exhibition with frequency and percent score in Sesame accessions

\begin{tabular}{|c|c|c|c|c|c|}
\hline $\begin{array}{l}\text { Sl. } \\
\text { No. }\end{array}$ & Descriptor & Sub descriptor & Score & $\begin{array}{c}\text { Frequency } \\
\text { observed }\end{array}$ & $\begin{array}{l}\text { Percent } \\
\text { score }\end{array}$ \\
\hline \multicolumn{6}{|c|}{ Plant descriptor } \\
\hline 1 & Plant growth type & $\begin{array}{l}\text { Indeterminate } \\
\text { Determinate }\end{array}$ & $\begin{array}{l}1 \\
2\end{array}$ & $\begin{array}{l}266 \\
454\end{array}$ & $\begin{array}{l}36.9 \\
63.1\end{array}$ \\
\hline 2 & Plant growth habit & $\begin{array}{l}\text { Prostrate } \\
\text { Semi-erect } \\
\text { Erect }\end{array}$ & $\begin{array}{l}1 \\
2 \\
3\end{array}$ & $\begin{array}{l}154 \\
308 \\
258\end{array}$ & $\begin{array}{l}21.4 \\
42.7 \\
35.9\end{array}$ \\
\hline 3 & Stem hairiness & $\begin{array}{l}\text { Absent } \\
\text { Sparse } \\
\text { Dense }\end{array}$ & $\begin{array}{l}1 \\
3 \\
5\end{array}$ & $\begin{array}{l}194 \\
377 \\
149\end{array}$ & $\begin{array}{l}27.0 \\
52.3 \\
20.7\end{array}$ \\
\hline 4 & Branching pattern & $\begin{array}{l}\text { Non branching } \\
\text { Basal branching } \\
\text { Top branching } \\
\text { Other }\end{array}$ & $\begin{array}{l}0 \\
1 \\
2 \\
3\end{array}$ & $\begin{array}{c}66 \\
371 \\
279 \\
04\end{array}$ & \begin{tabular}{c|}
9.1 \\
51.6 \\
38.8 \\
0.5
\end{tabular} \\
\hline \multicolumn{6}{|c|}{ Leaf descriptor } \\
\hline 5 & Leaf hairiness & $\begin{array}{l}\text { Glabrous } \\
\text { Weak } \\
\text { Medium } \\
\text { Strong }\end{array}$ & $\begin{array}{l}0 \\
3 \\
5 \\
7\end{array}$ & $\begin{array}{c}168 \\
348 \\
161 \\
43\end{array}$ & \begin{tabular}{r|}
23.3 \\
48.3 \\
22.4 \\
6.0
\end{tabular} \\
\hline 6 & Leaf shape & $\begin{array}{l}\text { Linear } \\
\text { Lanceolate } \\
\text { Elliptic } \\
\text { Ovate } \\
\text { Narrowly cordate }\end{array}$ & $\begin{array}{l}1 \\
2 \\
3 \\
4 \\
5\end{array}$ & $\begin{array}{l}461 \\
233 \\
23 \\
03 \\
00\end{array}$ & $\begin{array}{c}64.0 \\
32.4 \\
3.2 \\
0.4 \\
0.0\end{array}$ \\
\hline 7 & Basal leaf margin & $\begin{array}{l}\text { Entire } \\
\text { Serrate } \\
\text { Dentate }\end{array}$ & $\begin{array}{l}1 \\
2 \\
3\end{array}$ & $\begin{array}{l}330 \\
144 \\
246\end{array}$ & $\begin{array}{l}45.8 \\
20.0 \\
34.2\end{array}$ \\
\hline 8 & $\begin{array}{l}\text { Lobe incision of basal } \\
\text { leaf }\end{array}$ & $\begin{array}{l}\text { Absent } \\
\text { Weak } \\
\text { Medium } \\
\text { Strong }\end{array}$ & $\begin{array}{l}0 \\
3 \\
5 \\
7\end{array}$ & $\begin{array}{c}339 \\
225 \\
139 \\
17\end{array}$ & $\begin{array}{c}47.0 \\
31.3 \\
19.3 \\
2.4\end{array}$ \\
\hline 9 & Petiole colour & $\begin{array}{l}\text { Green } \\
\text { Greenish purple } \\
\text { Purple } \\
\text { Pink }\end{array}$ & $\begin{array}{l}1 \\
2 \\
3 \\
4\end{array}$ & $\begin{array}{c}714 \\
04 \\
02 \\
00\end{array}$ & $\begin{array}{c}99.2 \\
0.5 \\
0.3 \\
0.0\end{array}$ \\
\hline \multicolumn{6}{|c|}{ Inflorescence descriptor } \\
\hline 10 & Number of flowers per & One & 1 & 624 & 86.7 \\
\hline
\end{tabular}




\begin{tabular}{|c|c|c|c|c|c|}
\hline & leaf axil & More than one & 2 & 96 & 13.3 \\
\hline 11 & Corolla hairiness & $\begin{array}{l}\text { Absent } \\
\text { Sparse } \\
\text { Dense }\end{array}$ & $\begin{array}{l}1 \\
3 \\
5\end{array}$ & $\begin{array}{l}276 \\
280 \\
164\end{array}$ & $\begin{array}{l}38.3 \\
38.9 \\
22.8\end{array}$ \\
\hline 12 & Exterior corolla colour & $\begin{array}{l}\text { White } \\
\text { White with pink shading } \\
\text { White with deep pink } \\
\text { shading } \\
\text { Pink } \\
\text { Light violet } \\
\text { Dark violet } \\
\text { Purple } \\
\text { Red } \\
\text { Light maroon } \\
\text { Other }\end{array}$ & $\begin{array}{c}4 \\
5 \\
6 \\
7 \\
8 \\
9 \\
99\end{array}$ & $\begin{array}{c}281 \\
196 \\
70 \\
\\
150 \\
13 \\
09 \\
01 \\
00 \\
00 \\
00\end{array}$ & \begin{tabular}{|c|}
39.0 \\
27.2 \\
9.8 \\
\\
20.8 \\
1.80 \\
1.3 \\
0.1 \\
0.0 \\
0.0 \\
0.0
\end{tabular} \\
\hline 13 & Interior corolla colour & $\begin{array}{l}\text { White } \\
\text { White with pink shading } \\
\text { White with deep pink } \\
\text { shading } \\
\text { Pink } \\
\text { Light violet } \\
\text { Dark violet } \\
\text { Purple } \\
\text { Red } \\
\text { Light maroon } \\
\text { Other }\end{array}$ & $\begin{array}{c}4 \\
5 \\
6 \\
7 \\
8 \\
9 \\
99\end{array}$ & $\begin{array}{c}279 \\
187 \\
72 \\
\\
153 \\
13 \\
15 \\
01 \\
00 \\
00 \\
00\end{array}$ & \begin{tabular}{|l|}
38.8 \\
26.0 \\
10.0 \\
\\
21.2 \\
1.8 \\
2.1 \\
0.1 \\
0.0 \\
0.0 \\
0.0
\end{tabular} \\
\hline \multicolumn{6}{|c|}{ Capsule descriptor } \\
\hline 14 & $\begin{array}{l}\text { Number of locules per } \\
\text { capsule }\end{array}$ & $\begin{array}{l}\text { Four } \\
\text { Six } \\
\text { Eight } \\
\text { Mixed }\end{array}$ & $\begin{array}{l}1 \\
2 \\
3 \\
4\end{array}$ & $\begin{array}{c}689 \\
10 \\
10 \\
11\end{array}$ & $\begin{array}{r}95.7 \\
1.4 \\
1.4 \\
1.5\end{array}$ \\
\hline 15 & $\begin{array}{l}\text { Bicarpellate capsule } \\
\text { shape }\end{array}$ & $\begin{array}{l}\text { Tapered at apex } \\
\text { Narrow oblong } \\
\text { Broad oblong } \\
\text { Square }\end{array}$ & $\begin{array}{l}1 \\
2 \\
3 \\
4\end{array}$ & $\begin{array}{c}52 \\
343 \\
211 \\
114\end{array}$ & $\begin{array}{c}7.2 \\
47.6 \\
29.4 \\
15.8\end{array}$ \\
\hline 16 & Capsule arrangement & $\begin{array}{l}\text { Monocapsular } \\
\text { Multicapsular }\end{array}$ & $\begin{array}{l}1 \\
2\end{array}$ & $\begin{array}{c}680 \\
40\end{array}$ & $\begin{array}{r}94.4 \\
5.6\end{array}$ \\
\hline 17 & Capsule hairiness & $\begin{array}{l}\text { Glabrous } \\
\text { Sparse } \\
\text { Medium } \\
\text { Strong }\end{array}$ & $\begin{array}{l}0 \\
3 \\
5 \\
7\end{array}$ & $\begin{array}{c}249 \\
228 \\
160 \\
83\end{array}$ & $\begin{array}{l}34.6 \\
31.7 \\
22.2 \\
11.5\end{array}$ \\
\hline 18 & $\begin{array}{l}\text { Colour of dry Capsule } \\
\text { (Sun dried) }\end{array}$ & $\begin{array}{l}\text { Green } \\
\text { Straw/yellow } \\
\text { Brown/tan } \\
\text { Purple }\end{array}$ & $\begin{array}{l}1 \\
2 \\
3 \\
4\end{array}$ & $\begin{array}{c}26 \\
299 \\
355 \\
00\end{array}$ & $\begin{array}{c}3.6 \\
41.5 \\
49.3 \\
0.0\end{array}$ \\
\hline
\end{tabular}




\begin{tabular}{|c|c|c|c|c|c|}
\hline 19 & Type of capsule break & $\begin{array}{l}\text { Short } \\
\text { Long } \\
\text { Curved } \\
\text { Cleft } \\
\text { Other }\end{array}$ & $\begin{array}{c}1 \\
2 \\
3 \\
4 \\
99\end{array}$ & $\begin{array}{c}191 \\
209 \\
136 \\
144 \\
00\end{array}$ & $\begin{array}{c}26.5 \\
29.0 \\
18.9 \\
20.0 \\
0.0\end{array}$ \\
\hline \multicolumn{6}{|c|}{ Seed descriptor } \\
\hline 20 & Seed coat colour & $\begin{array}{l}\text { White } \\
\text { Cream } \\
\text { Beige } \\
\text { Light brown } \\
\text { Medium brown } \\
\text { Dark brown } \\
\text { Brick red } \\
\text { Tan } \\
\text { Olive } \\
\text { Grey } \\
\text { Dull black } \\
\text { Bright black } \\
\text { Other }\end{array}$ & $\begin{array}{c}1 \\
2 \\
3 \\
4 \\
5 \\
6 \\
7 \\
8 \\
9 \\
10 \\
11 \\
12 \\
99\end{array}$ & $\begin{array}{c}02 \\
66 \\
190 \\
118 \\
124 \\
126 \\
05 \\
00 \\
00 \\
02 \\
33 \\
14 \\
00\end{array}$ & \begin{tabular}{|c|}
0.2 \\
9.2 \\
26.3 \\
16.3 \\
17.2 \\
17.5 \\
0.7 \\
0.0 \\
0.0 \\
0.2 \\
4.6 \\
1.2 \\
0.0
\end{tabular} \\
\hline \multicolumn{6}{|c|}{ Disease score } \\
\hline 1. & Bacterial leaf blight & $\begin{array}{l}\text { No infection (Immune) } \\
\text { Resistant } \\
\text { Moderately resistant } \\
\text { Moderately susceptible } \\
\text { Susceptible } \\
\text { Highly susceptible }\end{array}$ & $\begin{array}{l}0 \\
1 \\
2 \\
3 \\
4 \\
5\end{array}$ & $\begin{array}{c}00 \\
00 \\
09 \\
513 \\
189 \\
09\end{array}$ & $\begin{array}{c}0.0 \\
0.0 \\
1.3 \\
71.3 \\
26.3 \\
1.3\end{array}$ \\
\hline 2. & Cercospora leaf spot & $\begin{array}{l}\text { No infection (Immune) } \\
\text { Resistant } \\
\text { Moderately resistant } \\
\text { Moderately susceptible } \\
\text { Susceptible } \\
\text { Highly susceptible }\end{array}$ & $\begin{array}{l}0 \\
1 \\
2 \\
3 \\
4 \\
5\end{array}$ & $\begin{array}{c}00 \\
00 \\
00 \\
67 \\
606 \\
47\end{array}$ & $\begin{array}{c}00 \\
00 \\
00 \\
9.3 \\
84.2 \\
6.5\end{array}$ \\
\hline
\end{tabular}


Table.2 Variability for morphological traits and promising lines identified for economic importance

\begin{tabular}{|c|c|c|c|c|}
\hline Character & Min & Max & Mean & Promising accessions \\
\hline Days to maturity & 77 & 106 & 86.2 & $\begin{array}{l}\text { IC0131801, IC0049260, IC0259397, } \\
\text { IC0044245, IC0325998, IC0131755, } \\
\text { IC0132349, IC0204834, IC0205314, } \\
\text { IC0268360, IC0205492, IC0325840, } \\
\text { IC0325969, IC0326040 and } \\
\text { EC0043760 }\end{array}$ \\
\hline Plant height (cm) & 25.3 & 135.7 & 72.1 & $\begin{array}{l}\text { IC0014143, EC0044247, IC0023288, } \\
\text { IC0041953, EC0133857, IC0021625, } \\
\text { IC0023298, IC0016243, EC0044246, } \\
\text { IC0132103, IC0021690, IC0131970, } \\
\text { IC0042459, IC0326040 and } \\
\text { IC0127279 }\end{array}$ \\
\hline $\begin{array}{l}\text { Number of } \\
\text { branches }\end{array}$ & 1.0 & 8.7 & 3.7 & $\begin{array}{l}\text { IC0011274, IC0350397, IC0127294, } \\
\text { IC0595438, IC0249016, IC0014173, } \\
\text { IC0024207, IC0041910, IC0041951, } \\
\text { IC0041954, IC0041977, IC0043014, } \\
\text { IC0110789, IC0127265 and } \\
\text { IC0131880 }\end{array}$ \\
\hline $\begin{array}{l}\text { Number of } \\
\text { capsules }\end{array}$ & 0.7 & 83.0 & 18.7 & $\begin{array}{l}\text { IC0014143, IC0127279, IC0023298, } \\
\text { IC0021625, IC0023320, IC0127265, } \\
\text { IC0041953, IC0016243, IC0021687, } \\
\text { IC0132103, IC0132047, IC0014146, } \\
\text { IC0042459, IC0014302 and } \\
\text { IC0127294 }\end{array}$ \\
\hline $\begin{array}{l}1000 \text { seed weight } \\
\text { (g) }\end{array}$ & 1.0 & 3.0 & 2.1 & $\begin{array}{l}\text { IC0014125, IC0001423, IC0014760, } \\
\text { IC0043654, IC0021690, IC0132018, } \\
\text { IC0132055, IC0132125, EC0089112, } \\
\text { IC0014117, IC0014131, IC0024212, } \\
\text { IC0043087, IC0132047 and } \\
\text { IC0132052 }\end{array}$ \\
\hline
\end{tabular}


Table.3 Accessions performing multiple traits in Sesame germplasm

\begin{tabular}{|c|c|c|c|c|c|c|c|c|c|c|c|c|c|c|c|c|c|c|c|c|c|c|c|c|}
\hline \multirow[b]{2}{*}{ Accession } & \multicolumn{2}{|c|}{$\begin{array}{c}\text { Plant } \\
\text { characters }\end{array}$} & \multicolumn{2}{|c|}{$\begin{array}{c}\text { Stem } \\
\text { characters }\end{array}$} & \multicolumn{3}{|c|}{$\begin{array}{c}\text { Leaf } \\
\text { characters }\end{array}$} & \multicolumn{3}{|c|}{$\begin{array}{c}\text { Inflorescence } \\
\text { characters }\end{array}$} & \multicolumn{6}{|c|}{ Capsule characters } & \multicolumn{5}{|c|}{ Quantitative traits } & \multirow{2}{*}{\begin{tabular}{|c|} 
Seed \\
Character \\
S \\
SC \\
\end{tabular}} & \multicolumn{2}{|c|}{$\begin{array}{l}\text { Disease } \\
\text { Score }\end{array}$} \\
\hline & PGT & PGH & SH & BP & LH & BLM & PC & FLA & $\mathbf{C H}$ & ECC & LOC & BCS & CPA & CPH & CDC & TCB & DM & $\mathbf{P H}$ & NB & NC & TW & & BLB & CLS \\
\hline IC0350397 & & & & & 7 & 2 & & & 5 & 3 & & 2 & 1 & & & & 88 & 88.7 & 7.7 & & & 3 & 2 & 4 \\
\hline IC0325998 & & 2 & & 2 & 7 & & & & & & 2 & & & 7 & 2 & & 79 & & & & 2.4 & 2 & 2 & 4 \\
\hline IC0326040 & & & 3 & 2 & & 3 & & 1 & 5 & 2 & 1 & & 1 & 7 & & 2 & 80 & & & & & 3 & 2 & 4 \\
\hline IC0024207 & 2 & 2 & & & 5 & 3 & 2 & 1 & & & 1 & & 1 & & 3 & & 97 & & & & & 6 & 2 & 4 \\
\hline IC0127265 & & & & & & & & & & & 1 & 2 & 1 & & & 1 & 84 & 76.0 & 6.0 & 52 & & 6 & 2 & 4 \\
\hline IC0326111 & & & & 2 & & 3 & & & & & 3 & & 1 & & 2 & & 87 & 101.3 & & & & 4 & 2 & 4 \\
\hline IC0331712 & & & & & & 3 & & & 5 & & 1 & 4 & 1 & 7 & 2 & 1 & 87 & 89.3 & 5.3 & 39 & & 4 & 2 & 4 \\
\hline IC0331713 & & & & & & 3 & & & 5 & & 1 & 2 & 1 & 5 & 2 & & 86 & 94.0 & 4.7 & 24 & & 5 & 2 & 4 \\
\hline IC0021690 & & 3 & 5 & 2 & 7 & 2 & & 2 & 5 & & & & & 7 & 2 & & & 107.3 & & & & & & \\
\hline EC0089111 & 2 & 3 & & & & & & 2 & & 5 & & 2 & 2 & & 2 & & & 36.7 & & & 1.7 & 3 & 3 & 4 \\
\hline IC0014143 & & 3 & & 2 & & & & 2 & & & & & & & 2 & & & 135.7 & 5.0 & 83 & & 3 & & \\
\hline IC0011274 & & & & 2 & & 2 & & 1 & & & 1 & 2 & 1 & & 3 & 1 & & 62.3 & 8.7 & 37 & & 4 & & \\
\hline IC0014125 & 2 & 3 & & & & & & 2 & & 5 & & 2 & & & & & & 68.0 & & 30 & 3.0 & 5 & & \\
\hline IC0131740 & & 3 & & 2 & 7 & 2 & 3 & & 5 & & & & & & 2 & 1 & & & & & & 6 & 3 & 4 \\
\hline IC0014760 & 2 & & 5 & & & 3 & & & & & & 2 & & & & & & 79.3 & 4.3 & 38 & 2.9 & & 3 & 4 \\
\hline EC0043708 & 2 & 3 & & & & & & & & & & 2 & & & & & & 82.7 & & 24 & 2.2 & 1 & & \\
\hline IC0049260 & 2 & 3 & 5 & & & 3 & & & & & & & & & 2 & & 78 & & & & 2.1 & & 3 & 4 \\
\hline IC0131801 & 2 & & & & 7 & & & & & & & & & & 2 & & 77 & & & & & & & \\
\hline IC0259397 & & & & 2 & & & & & 5 & & & & & & & & 78 & & & & 2.1 & & & \\
\hline IC0370503 & & & & & & & & & & 4 & 1 & & 1 & 7 & 2 & 1 & 81 & & & & & 3 & 2 & \\
\hline IC0127279 & & & 5 & & & & & & & & & 2 & & & & & & 99.0 & & 79 & & 2 & 3 & 3 \\
\hline & $\begin{array}{l}\text { PGT: Plan } \\
\text { LH: Leaf } \\
\text { CH: Coro } \\
\text { CPA: Cap } \\
\text { DM: Days } \\
\text { TW: } 1000\end{array}$ & $\begin{array}{l}\text { growth typ } \\
\text { iriness } \\
\text { hairiness } \\
\text { le arrange } \\
\text { o maturity } \\
\text { eed weigh }\end{array}$ & & & $\begin{array}{l}\text { PGH: Pl } \\
\text { BLM: B } \\
\text { ECC: Ex } \\
\text { CPH: C. } \\
\text { PH: Plan } \\
\text { SC: Seed }\end{array}$ & $\begin{array}{l}\text { t growth } \mathrm{h} \\
\text { liteaf ma } \\
\text { tior corollil } \\
\text { ule hairin } \\
\text { eight (cm } \\
\text { oat colour }\end{array}$ & & & $\begin{array}{l}\text { Stem ha } \\
\text { Petiole c c } \\
\text { No. of } \\
\text { Colour } \\
\text { No. of br } \\
\text { : Bacteri }\end{array}$ & $\begin{array}{l}\text { ur } \\
\text { cules per ca } \\
\text { dry Capsu } \\
\text { ches } \\
\text { leaf blight }\end{array}$ & $\begin{array}{ll} & \text { BP: } \\
\text { FLA } & \text { FCS } \\
\text { le } & \text { TCB } \\
\text { TC: } & \text { CLS }\end{array}$ & $\begin{array}{l}\text { ranching } \\
\text { No. of flo } \\
\text { Bicarpella } \\
\text { Type of c. } \\
\text { oo. of caps } \\
\text { Cercospor }\end{array}$ & $\begin{array}{l}\text { attern } \\
\text { vers per le: } \\
\text { e capsule s } \\
\text { psule break } \\
\text { les/plant } \\
\text { leaf spot }\end{array}$ & & & & & & & & & & & \\
\hline
\end{tabular}


Fig.1a Frequency distribution of different morphological traits in Sesame
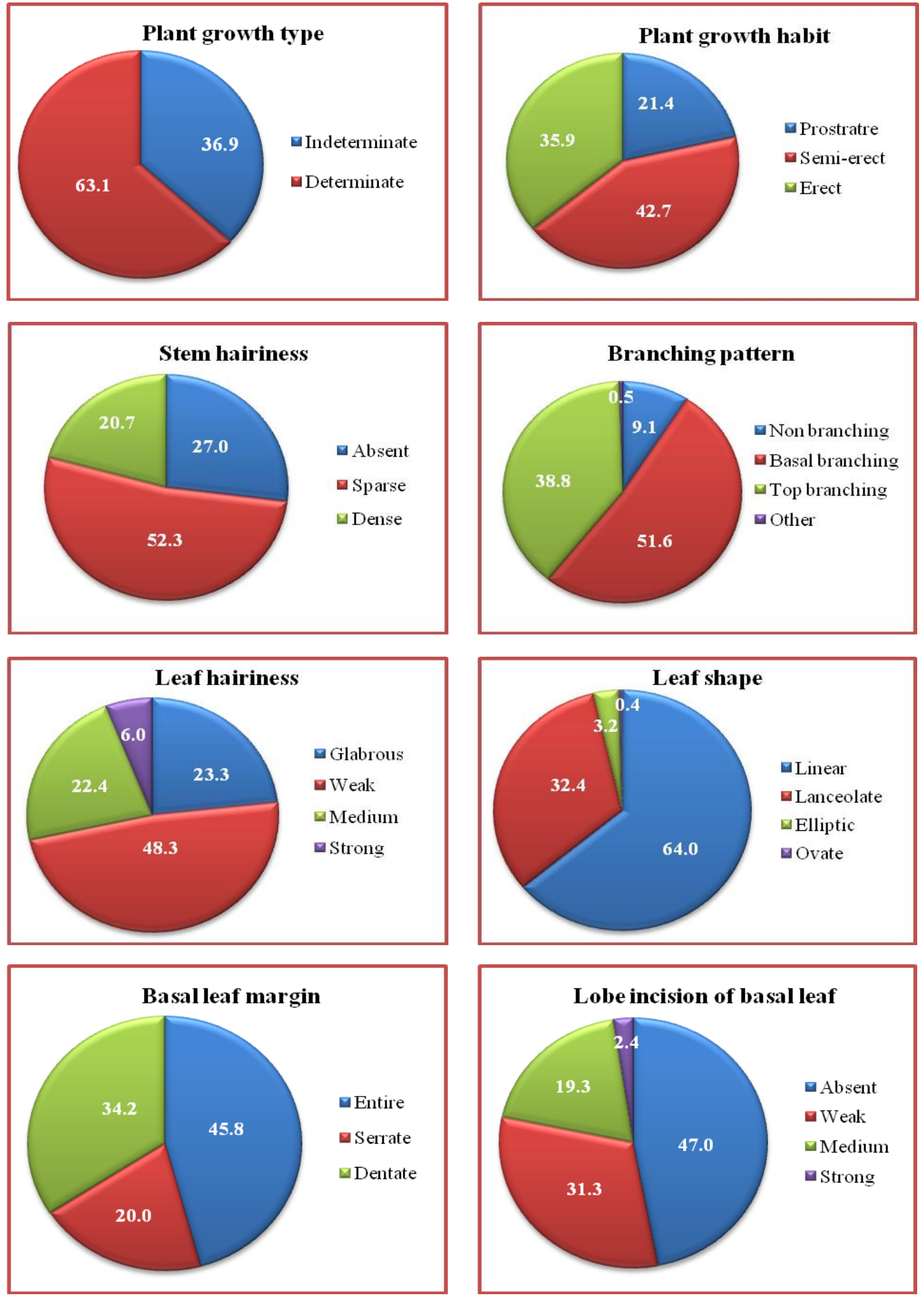
Fig.1b Frequency distribution of different morphological traits in Sesame (contd..)
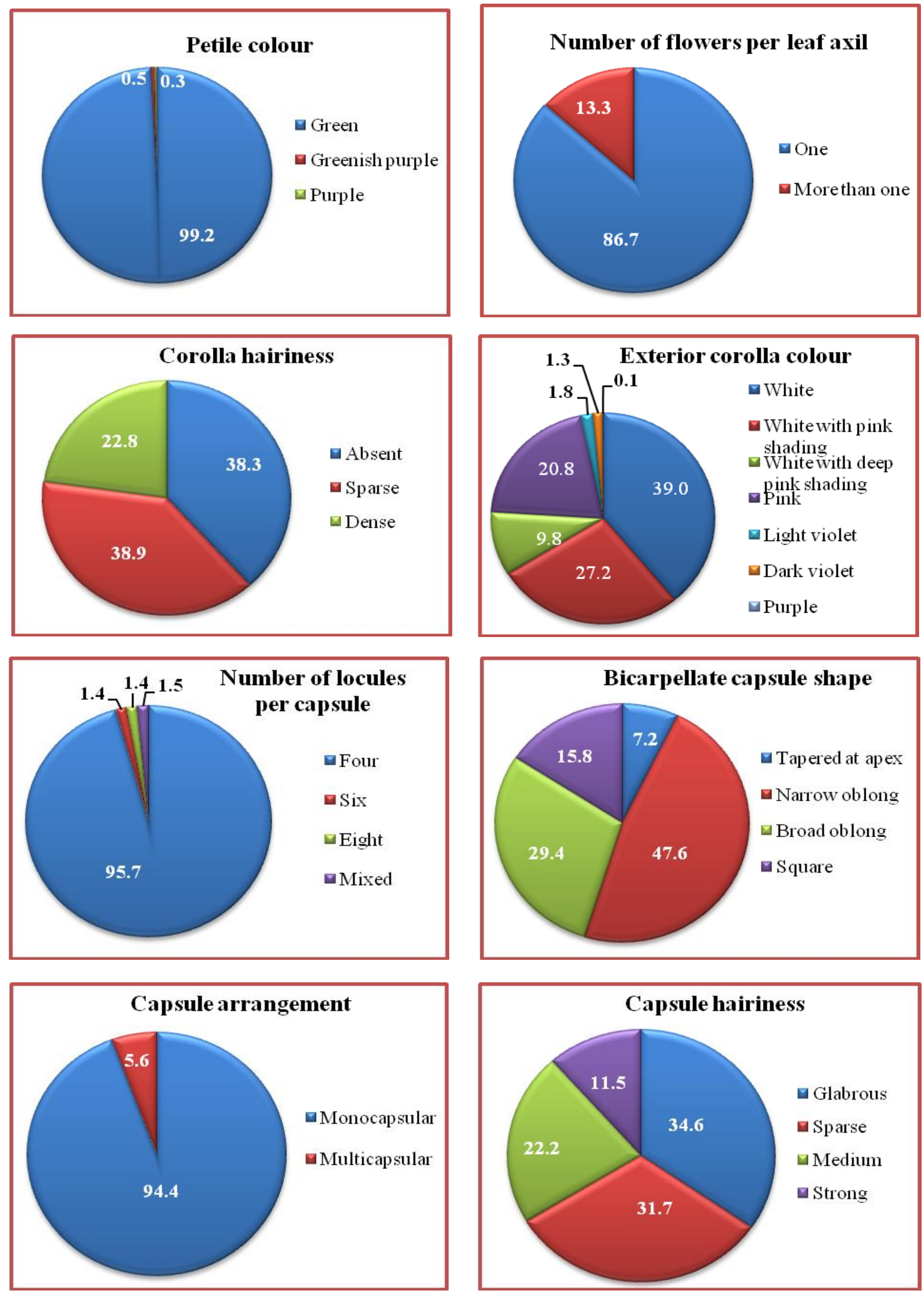
Fig.1c Frequency distribution of different morphological traits in Sesame
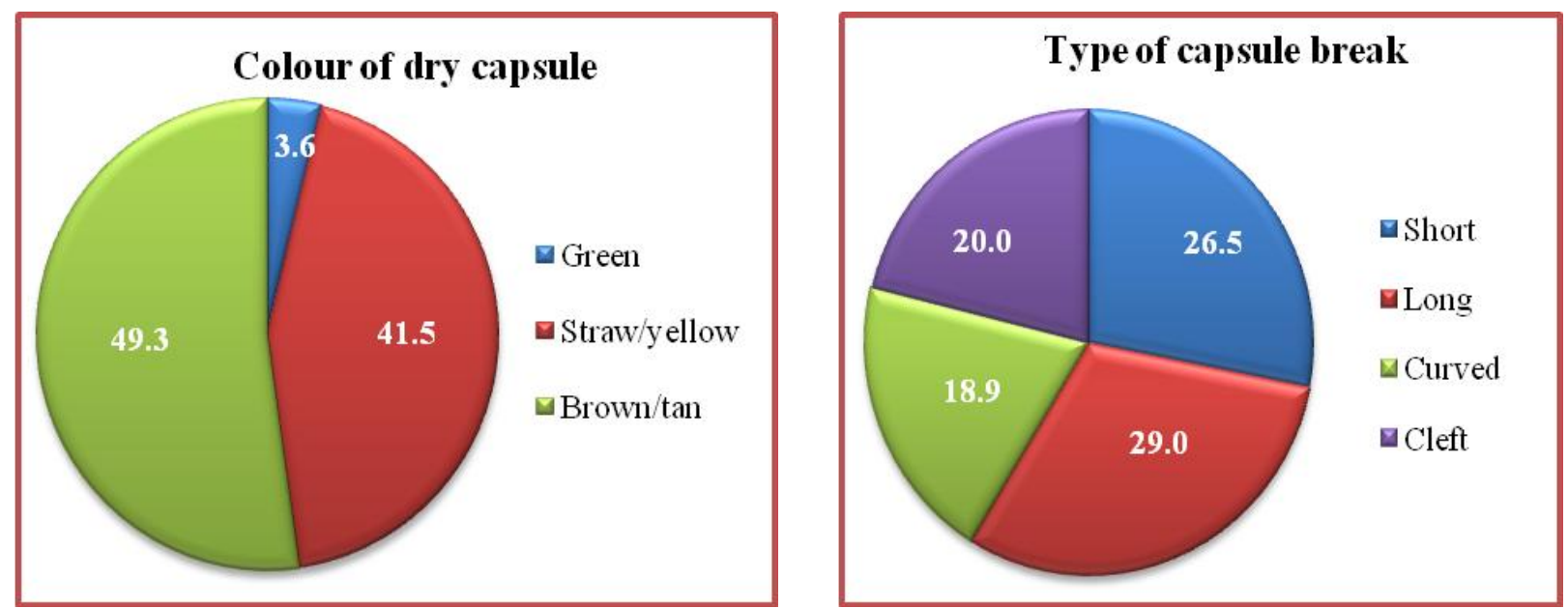

Fig.2 Frequency distribution of disease reaction in Sesame

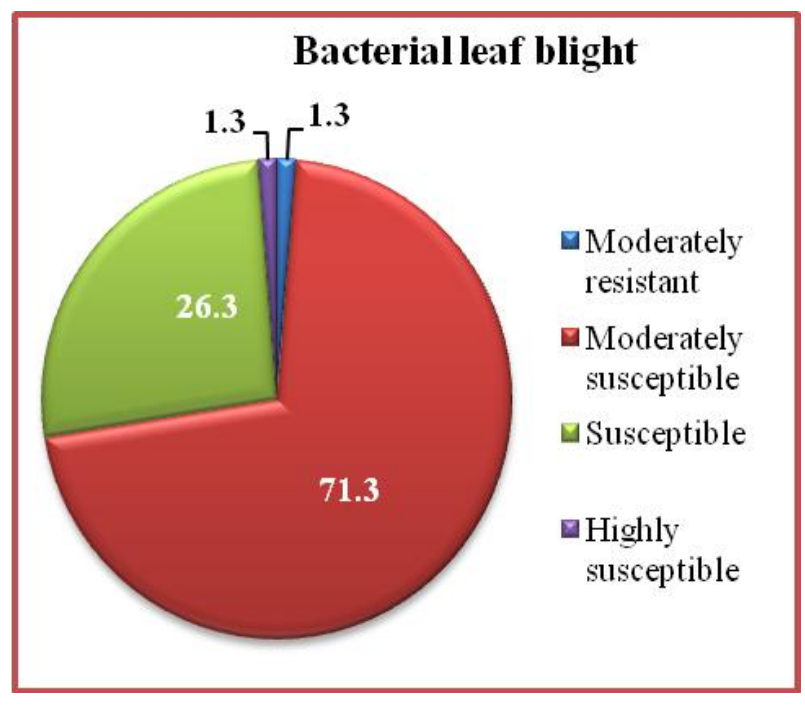

\section{Seed descriptor}

Seed coat colour was beige $(26.3 \%)$ followed by dark brown $(17.5 \%)$, medium brown (17.2 $\%)$, dull black (4.6\%) and cream colour $(9.2$ $\%)$. Most of farmers prefer white to brown seed colour. However, creamy white was in higher frequency as this is the most favored color among the farmers. Similar reports shown by Suhasini (2006) in Sesame. A wide range of variation beige, dark brown, medium brown, cream colour was observed against reported white, black and brown. Research in

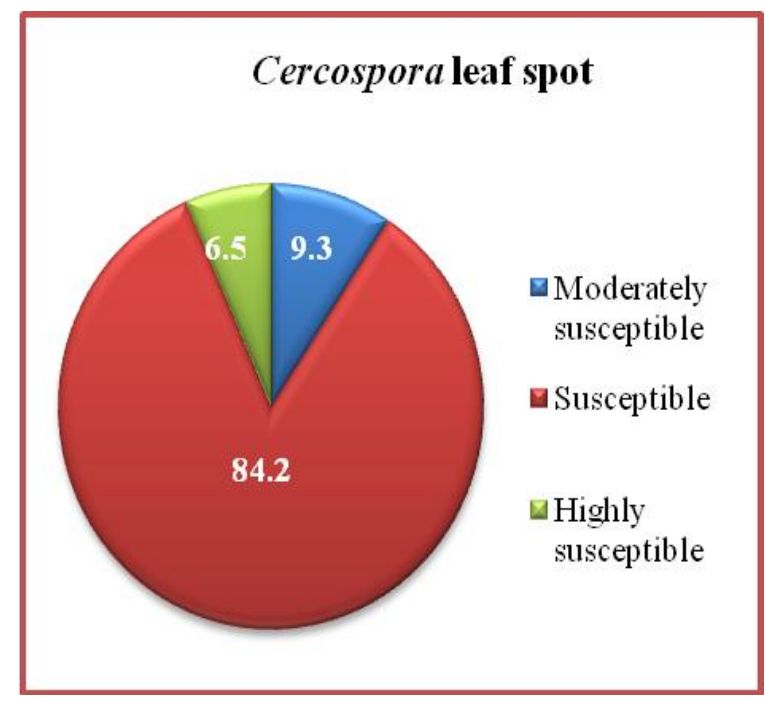

Sesame outlined by earlier breeders due to under digenic control with several confusing segregants beyond plausible explanation (Baydar and Turgut, 2000; Falusi, 2007). Zhang et al., (2013) studied using high density linkage map analysed the genetic segregation and QTL for sesame seed colour and showed that two major genes with additive dominant epistatic effects along with polygenes were responsible for controlling seed coat colour. The highest polymorphism was observed from white to black through all intermediate colours. 


\section{Disease score}

Among the accessions evaluated for foliar diseases, only $1.3 \%$ of accessions rated grade 2 for bacterial leaf blight followed by $71.3 \%$ of grade 3 and $9.3 \%$ of accessions recorded grade 3 for Cercospora leaf spot indicating moderately susceptible genotypes in the studied set under protected situation. Totally nine accessions viz., IC0350397, IC0370503, IC0325998, IC0326040, IC0024207, IC0127265, IC0326111, IC0331712 and IC0331713 exhibited moderately resistant to bacterial leaf blight and graphical representation shown in Figure 2.

\section{Quantitative characters}

Evaluation for yield and its attributing characters was carried out for identifying elite promising lines as shown in Table 2. For early maturing (77 days) accessions are IC0131801, IC0049260, IC0259397, IC0044245 and IC0325998. For plant height $135.7 \mathrm{~cm}$ with average of $72 \mathrm{~cm}$ accessions are IC0014143, EC0044247, IC0023288, IC0041953 and EC0133857. Higher number of branches exhibited in IC0011274, IC0350397, IC0127294, IC0595438 and IC0249016 and more number of capsule accessions viz., IC0014143, IC0127279, IC0023298, IC0021625 and IC0023320. Test weight of 1000 seeds promising accessions are IC0014125, IC0001423, IC0014760, IC0043654 and IC0021690 indicating bold seeds.

\section{Identification of germplasm accessions for multi-traits}

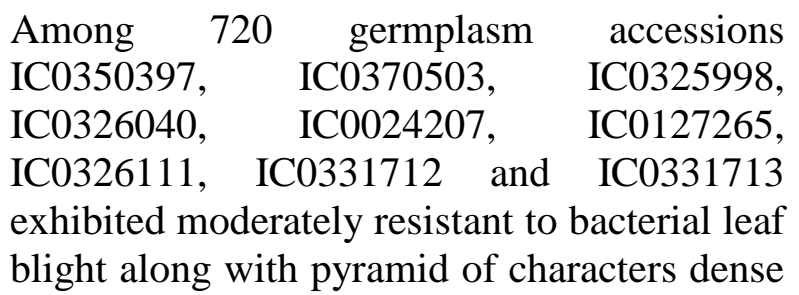

hairiness on capsules, monocapsular arrangement with four locules per capsule with short capsule break and dentate leaf margin on basal leaf with early maturing accessions as shown in Table 3.

Accession IC0021690 showing multiple traits for erect plant growth habit with dense hairiness, top branching pattern on stem. Regarding leaf characters strong hairs on leaf and serrated type leaf margin. More than one flower per leaf axil and dense corolla hairiness for inflorescence characters. Profuse hairiness on capsules and dry capsules color was observed to be straw yellow with 107.3 $\mathrm{cm}$ plant height.

Accession EC0089111 showed determinate growth with erect growth habit for plant characters with more than one flower per leaf axil and light violet exterior corolla colour, narrow oblong bicarpellate capsule shape having straw yellow colour for dry capsules with dwarf type having $36.7 \mathrm{~cm}$ of plant height with 87 days of maturity with beige seed colour.

Accession IC0014143 exhibited erect plant growth habit with more than two flowers per leaf axil exhibiting straw yellow colour for dry capsules with highest number of capsules per plant (83) with highest plant height of $135.7 \mathrm{~cm}$. IC0011274 accession was observed to have higher number of branches (8.7) with serrated leaf margin, narrow oblong bicarpellate capsule shape with short type of capsule break.

Accession IC0014125 showed highest test weight of $3.0 \mathrm{~g}$ with determinate growth with erect growth habit for plant characters and having more than 2 flowers per leaf axil with light violet exterior corolla colour and bicarpellate capsule shape was narrow oblong. IC0131740 accession exhibited erect growth habit with dense hairs on leaves, 
serrated basal leaf margin purple colour petiole colour showing dense hairiness on corolla with straw yellow dry colour capsules with short capsule break with dark brown seed colour.

IC0014760 germplasm accession exhibited determinate growth type, dense hairs on stem, dentate basal leaf margin, narrow oblong bicarpellate capsule shape with higher test weight of $2.9 \mathrm{~g}$.

Accession EC0043708 showed determinate growth with erect growth habit for plant characters, narrow oblong bicarpellate capsule shape with plant height of $82.7 \mathrm{~cm}$ and test weight of $2.2 \mathrm{~g}$ bearing white seed colour. IC0325998 accession showed profuse leaf hairs for leaf charcter and for capsule characters exhibited six locules with strong hairiness on capsule, straw yellow dry colour capsules with 79 days of early maturity, test weight of $2.4 \mathrm{~g}$ bearing cream colour seeds.

Accessions IC0049260, IC0131801 and IC0259397 are early maturing types with 7778 days with determinate plant growth type.

In conclusions, majority of sesame accessions were found to possess determinate growth type with semi-errect habit bearing sparse hairiness on stem with basal branching pattern with respect to plant descriptors. Abundance of accessions exhibited Linear leaf shape, entire leaf margin with green petiole colour for leaf descriptors. Regarding inflorescence descriptors majority of accessions recorded one flower per leaf axil bearing sparse hairiness with white corolla colour exterior and interior respectively. With respect to capsule descriptor higher frequency of accessions revealed four locules per capsule, monocapsular arrangement with brownish pattern of seed coat colour form light to medium to dark tint with moderately resistant to susceptible accessions for bacterial leaf blight and Cercospora leaf spot respectively. Accessions with pyramid of desired characters viz., IC0350397, IC0370503, IC0325998, IC0326040, IC0024207, IC0127265, IC0326111, IC0331712 and IC0331713 could be used for future crop improvement programmes for biotic and abiotic stress. Above study revealed the distinct characters of sesame accessions and noticed the morphological variations due to genetic make-up and could be better exploited by breeders in selections based on specific requirement for crop improvement programmes and this is highly useful investigation for varietal identification and conservation. The identified DUS traits will serve as a marker in selection process which are less influenced by environmental fluctuations and are easily scorable and stable expression. They serve as diagnostic descriptors of germplasm accessions and hence useful to avoid mistakes in labelling, aid identification and minimize duplication in the germplasm and helps in the development of guidelines for varietal release and registaration of new varieties of sesame.

\section{Acknowledgement}

The authors are grateful to Project Coordinating Unit (Sesame and Niger), JNKVV, Jabalpur for providing germplasm accessions and financial support of this Research under Consortium Research Project on Biodiversity.

\section{References}

Anonymous. 2015. Annual progress report, Sesame and Niger, Project Coordinating Unit (Sesame and Niger), J.N.K.V.V. Campus, Jabalpur.

Baydar, H. and Turgut, I. 2000. Studies on genetics and breeding on Sesame (Sesamum indicum L.). Inheritance of the characters determining plant type. Turk. J. Biol. 24: 503-512. 
Bedigian, D. and Harlan, J.R. 1986. Evidence for cultivation of sesame in the ancient world. Economic Botany. 40: 137-154.

Bhagwat Singh, Rajani Bisen and Akanksha Tiwari. 2017. DUS Testing of Sesame (Sesamum indicum L.) varieties using Morphological Descriptors. Bull. Env. Pharmacol. Life Sci. 6(1) 2017 5-12

Debas, B. S., Mathur, P. N. and Pareek, S. K. 1994. Collection, Characterization and maintenance of plant genetic resources of millets, arid legumes, medicinal plants and aromatic plants. Ex-situ conservation of plant genetic resources. National Bureau of Plant Genetic Resources, ICAR, New Delhi-110012. pp. 72-80.

Deepthi, P., Shukla, C. S., Verma, K. P. and Siva Sankar Reddy, E. 2014. Yield assessment and influence of temperature and relative humidity on charcoal rot development in sesame (Sesamum indicum L.). The Bioscan. 9:193-195. Dep.Agric. India. Bot. 18: 127-147.

Ezilkumar, S. 1999. Studies on varietal identification in hybrids, parents and varieties in Cotton (Gossypium spp.). M.Sc. (Agri.) Thesis, Tamil Nadu Agricultural University, Coimbatore.

Falusi, O. 2007. Segregation of genes controlling in Sesame from Nigeria. Afr. J. Biotechnol. 6(24): 2780-2783.

Federer, W. T., 1956. Augmented (or hoonuiaku) designs. Hawaii. Plant Research, 2: 191-208.

Harshiya Banu, Jayarame Gowda and M. V. Channabyre Gowda. 2018. Characterization and identification of DUS traits in Reference set of Foxtail millet germplasm (Setaria italica (L.) Beauv.). Int.J.Curr.Microbiol.App.Sci. 7(12): 175-183

Jagadeesh Babu, C. S., Byre Gowda, M., Girish, G. and Gowda, T. K. S. 2008. Screening of Dolichos germplasm for pod borers and bruchids. Environment and Ecology. 26 (4c): 2288-2290.

Jain, S. K., Khare, D., Bhale, M. S. and Raut, N.D. 2002. Characterization of Mung bean varieties for verification of genetic purity. Seed Tech News. 32 (1): 200201.

Kashiram, 1930. Studies in Indian oil seed; The types of Sesamum indicum D.C. Mem.

Kwon, Y. S., Lee, J. M. and Yi, G. B. 2005. Use of SSR markers to complement tests of distinctiveness, uniformity, and stability (DUS) of pepper (Capsicum annuum L.) varieties. Molecules and Cells. 19(3): 428-435.

Liang, M. D., Gatarayiha, M. C. and Adandonon, A. 2006. Silicon use for pest control in agriculture: A review. Proc. S. Afr. Sug. Technol. Ass. 80: 278286.

Lombard, V., Baril, C. P., Dubreuil, P., Blouet, F. and Zhang, D. 2000. Genetic relationships and fingerprinting of markers to complement distinctness, uniformity and stability testing of rape (Brassica napus L.) varieties. Theoretical and Applied Genetics. 106(6): 1091- 1101.

Palakshappa, M. G., Parameshwarappa, S.G. and M. S. Lokesh. 2009. Integrated management of Cercospora leaf spot of sesame. In: National symposim on Integrated approach-In the sustainable management of plant diseases, October, 9-10,2009 at UAS, Dharwad, Karnataka. pp.75.

Prasad, R. 2002. Text Book of field Crops Production, Indian Council of Agricultural Research, New Delhi, p 821.

Sarita, K. Pandey, Arna Das and Tapash Dasgupta. 2013. Genetics of seed coat color in sesame (Sesamum indicum L.). African Journal of Biotechnology. 12 (42): 6061-6067

Shigeta, M. 1985. The genetic diversity of 
finger millet (Eleusine coracana, Gaertn.) and wild finger millet (sp. africana) M.Sc. (Agri) Thesis, Kyoto University. Japan.

Suhasini, K. S, 2006. Characterization of sesame genotypes through morphological, chemical and RAPD markers, M.Sc. thesis submitted to the university of agricultural sciences. Dharwad.

Tommasini, L., Batley, J. and Arnold, G. M. 2003. The development of multiplex simple sequence repeat (SSR). SABRAO Journal of Breeding and Genetics. 44 (2) 292-301.
Upadhyaya, H. D., Yadav, D., Dronavalli, N., Gowda, C. L. L. and Singh, S. 2010. Mini core germplasm collections for infusing genetic diversity in plant breeding programs. Electron. J. $\mathrm{Pl}$. Breed. 1 (4): 1294-1309.

Weiss, E. A. 1971. Castor, Sesame and Safflower, Leonard Hill Books, London, 311-355.

Zhang, H., Miao, H., Wei, L. Li, C., Zhao, R. and Wang, C. 2013. Genetic analysis and QTL mapping of Seed coat colour in Sesame (Sesamum indicum L.) PLoS ONE. 8: e 63898 doi 101371

\section{How to cite this article:}

Palakshappa, M. G., Harshiya Banu, S. G. Parmeshwarappa, Rajani Bisen, H. Nagappa and Pooja Holeyannavar. 2020. DUS Testing of Sesame (Sesamum indicum L.) Accessions Using Morphological Descriptors and Evaluation for Foliar Diseases of Sesame. Int.J.Curr.Microbiol.App.Sci. 9(01): 1837-1852. doi: https://doi.org/10.20546/ijcmas.2020.901.206 